\title{
Linking Biotechnology and Agricultural Biodiversity Resources in Holistic Strategy in West Africa
}

\author{
J. J. Baidu-Forson ${ }^{1 *}$ and R. Lewis-Lettington ${ }^{2}$ \\ ${ }^{1}$ Sub-Saharan Africa, Bioversity International, P. O. Box 30677, Nairobi 00100, Kenya \\ ${ }^{2}$ Global Coordination Office for Genetic Resources Policy Initiative (GRPI), Bioversity \\ International, P.O. Box 30677, Nairobi 00100, Kenya \\ *Corresponding author; E-mail: j.baidu-forson@cgiar.org
}

\begin{abstract}
Modern economic activities are heavily dependent on using diversity of biological resources. Africa has a wealth of biodiversity resources which, with the appropriate application of biotechnological tools for conservation and use, can serve as sources of wealth creation. Proper harnessing of the linkages between biotechnology and the diversity of biological resources is required to meet challenges of food security, health, poverty and wealth creation in West African countries. The paper explores some of the key applications of biotechnology for conservation of agricultural biodiversity resources, and considers the potential threat of biotechnology to diversity of genetic resources. It also explores complex issues that inform current policy debates. It concludes that Government support is required for the conservation and breeding of farmers' varieties, or landraces by public breeding programmes, and the design of private and public mechanisms to ensure that the pursuit of biotechnology does not compromise the diversity in biological resources. It would be strategic for West African countries to establish and explore beneficial linkages between the subregional genetic resources conservation initiative and biotechnology programmes. Some pertinent questions are raised on how to best manage the strategic interplay between biotechnology and diversity in agricultural biodiversity resources. The provision of adequate information is highlighted to inform decisions and choices based on the real value and potential risks of biotechnology.
\end{abstract}

\section{Introduction}

Biological diversity has contributed in many ways to the development of human culture, and mankind has in turn influenced biological diversity (FAO, 1999). In today's modern economy, agriculture, pharmaceuticals, traditional medicine, forestry, fisheries and tourism are heavily dependent on using a diversity of biological resources. AU/NEPAD High-Level Panel on Biotech-nology (2006) rightly recognized that one of the critical capabilities that exist in Africa is the wealth of biodiversity that can potentially serve as a resource for wealth creation with the aid of biotechnological tools. But how these resources are managed, particularly how they are conserved and used, has a profound effect, for better or for worse, on available diversity and the ecological services that sustain life. For example, destruction of habitats as a result of competing human needs has often resulted in the loss of numerous plant and animal species and the services they help provide. On the other hand, proper harnessing of the linkages between biotechnology and the diversity of the biological resources provides a good platform for conservation of the diversity required to meet challenges of food security, health, poverty, wealth creation and the furtherance of overall developmental goals of West African countries.

FAO (1993) defines agricultural biodiversity as the variety and variability of animals, plants and micro-organisms that are important to food and agriculture which result from the interaction between the environment, genetic resources and the management systems and practices used by people. Mulvany (1998) describes agricultural biodiversity as a vital subset of biodiversity comprising of varieties, breeds, species and agro-ecosystems that underpin universal food security and provide the genetic material needed for industry, agriculture and biotechnology. In agriculture, biotechnology has a potential role in enhancing agricultural productivity, food security and promoting the conservation, scientific characterization and use of genetic resources to improve human well-being. Therefore, as an integral part of the focus on developing a holistic biotechnology strategy for West African countries, the need to explore and incorporate the 
beneficial interplay between biotechnology and agricultural biodiversity resources cannot be overemphasized.

\section{Exploring the benefits of biotechnology for biodiversity}

Africa's wealth in its diversity of biological resources, generally, and agricultural biodiversity, in particular, is rapidly being lost in many ways, for instance, through genetic erosion. The continued loss of diversity in genetic materials needed for the improvement of yields or productivity, as well as for enhancing capacity to deal with diseases, pests and the vagaries of the weather cannot be ignored. Therefore, it is imperative that, in formulating a biotechnology strategy and plan of action, West African countries explore how to effectively use modern agricultural biotechnology tools to help stem the rapid loss of diversity in agriculturally useful materials.

Examples of biotechnological tools that are relevant to the conservation of genetic resources include in-vitro techniques, such as slow growth tissue culture, and cryopreservation (for long term conserva-tion). Cryopreservation of plant material represents a safe and cost-effective option for long-term conservation of germplasm of non-orthodox seed species, vegetatively-propagated species, and biotechnology products (Englemann, 2004). For example, cryopreservation of shoottips is the method of choice for the collection, safe movement and long-term conservation of seedless and, thus, vegetatively-propagated crops like banana (Musa spp.). Large scale cryopreservation of embryogenic cultures has been found to be essential for advanced forestry breeding programmes (Panis et al., 2004). Cryopreservation has been shown to improve the frequency of virus elimination - specifically cucumber mosaic virus and banana streak virus (Helliot et al., 2002).

A simple cryopreservation method is available for proliferating meristem cultures of banana (Musa spp.). The miniaturization of explants reduces space requirements and, consequently, labour costs for maintenance and transporting germplasm collections. Tissue culture systems allow the propagation of virus-free plant material with high multiplication rates using meristem culture in combination with thermotherapy. Biotechnology also has an important role or value through the use of molecular markers for prioritizing populations for conservation and for characterizing populations (e.g. fish stocks). The use of molecular marker techniques for characterization work and genetic mapping could enhance the growing of crops and other plants at the gene level.

The Consultative Group on International Agricultural Research (CGIAR) is investing heavily in biotechnological methods, through the Generation Challenge Programme, to use advances in molecular biology to harness the rich global heritage of plant genetic resources, and create a new generation of crops that meet the needs of resource-poor people. Better-performing crops improve rural livelihoods by increasing food security and income. For example, the International Rice Research Institute (IRRI) uses applications of biotechnology, in vitro culture of seedlings and the study of genetic diversity using a range of molecular markers. Understanding of the genome of one crop, like rice, can yield useful clues about others, such as wheat, barley and maize because there is a great similarity among the genomes of species in the same family. In such situations, isolating the desired gene in one plant for a particular key trait, such as plant height, offers valuable clues to the same trait in all the others in the same family (Devos \& Gale, 2002).

Effective use of agricultural biodiversity resources requires fundamental activities such as acquisition of good knowledge of species and genes through collection, characterization and documentation of the resources and their associated indigenous knowledge, and conservation and under-standing of the value of the genetic materials for use in applications that will improve the well-being of the human population. The relevant activities and the associated information to be generated are fundamental to a productive application of biotechnology to plants/species (both

West African Journal of Applied Ecology, Vol. 12, 2008 
introduced and underutilized indigenous plants) to resolve biotic and abiotic constraints, and/or increase productivity in the West African countries.

\section{Is biotechnology a threat to diversity?}

Discourse regarding the nature of the threats presented by modern biotechnologies is almost as complex as the technologies themselves. It would not be possible to cover all the perceived threats in this paper. However, a few illustrative cases are discussed. Biotechnology is sometimes seen as a tool encouraging privatization of resources. Fears are often expressed that biotechnology will reduce crop biodiversity and that the increasing privatization of crop genetics will hurt poor farmers and exacerbate hunger among the world's poor. Some controversies have revolved around property rights of private companies that will be motivated by the pursuit of biotechnology to invest in hitherto neglected food crops and the potential limitation to farmers' ability to save and use their own seed. In particular, the 'technology protection system' or the 'terminator technology' has provided a lightening rod for controversy and fears about the potential impacts of patents on society at the level of food production (Cox, 1988).

Widespread adoption of varieties carrying the 'technology protection system' will undermine the culture of farmer seed selection and conservation for planting or later exchange, and lead to crop uniformity and potential future vulnerability to biotic and abiotic stresses. Furthermore, biosafety concerns have been raised about sterility traits escaping beyond the boundaries of fields planted to protected varieties and infecting neighbouring fields of the same crop and their wild relatives through pollen produced by plants carrying the technology protection system. Doubts have been raised about the seriousness of these biosafety concerns because the natural outcrossing of some self-pollinated crops is only up to 5\% (Cox, 1988). The issue is that if a conventional farmer's variety is planted next to variety with a technology protection system, they must be in close proximity and there must be a similarity of flowering dates. Moreover, the likelihood of a trait being passed to another species is even lower because normally foreign pollen is unable to fertilize an unrelated species (Cox, 1988).

Environmental threats, through potential reduction in genetic diversity with potential long-term effects on food security, have also been highlighted. Instead of negating the use of biotechnology for seed production, there is rather an argument for strengthening local seed systems to counterbalance seed production through the use of biotechnology. Also, there is some doubt that the mere availability of new varieties using the technology protection system will limit farmers' ability and willingness to select, conserve and use their own seeds or develop their own locallyadapted varieties. In the light of all the above arguments, are there real negative outcomes that are the inevitable consequences of using biotech-nology? It may be the case that private sector pursuit of biotechnology, driven mainly by profitability and proprietary reasons, may move in the direction of narrowing diversity in agricultural biodi-versity resources. In this case, the public research system has a critical and valuable function to undertake in encouraging the conservation of local agricultural biodiversity resources for use in research or for direct use by farmers. This translates into govern-ment support for the conservation and breeding of farmers' usual varieties or landraces by public breeding programmes. At the ECOWAS level, the question should be what model private and public mechanisms (institutional, legal, policy, advocacy) can be devised and agreed on regionally to ensure that the pursuit of biotechnology does not compromise the diversity in the biological resources on which our people depend for food and agriculture.

\section{Policy issues}

Biotechnology policy is a complex field that, more accurately, refers to a range of policy issues of relevance to a family of, often only loosely related, technologies. For example, the issues that may arise in relation to the introduction of genetically modified organisms are unlikely to be

West African Journal of Applied Ecology, Vol. 12, 2008 
similar to those involved with the release of the products of micropropagation. As a result, the first step in any policy formulation process is to ensure that one is quite clear about what is being discussed and that there is a framework for these discussions that is flexible enough to accommodate multiple approaches for multiple technologies. Closely related to accurately defining the subject matter of discussions is the question of clearly stating the objectives of any policy. The relative weighting of factors involved with, and desired outcomes from, risk and benefit assessment in different fields is of fundamental importance in moulding any policy. Even after having established the basic parameters of any policy debate, biotechnology remains a complex field. For example, biosafety policy revolves around the classic regulatory balance of safety (of the environment, of livelihoods and of commercial options as the three most obvious factors) versus freedom of action but involves a scenario where there are multiple opinions, and considerable uncertainty, regarding almost all of the factors.

At a strategic level, this may involve consideration of the appropriate level of burden to place upon the providers and users of particular technologies relative to the range of perceived risks and benefits, actual and potential, involved with their activities. These burdens can be imposed upfront, in terms of authorisation requirements and procedures, or they can be downstream, focusing on liability and redress, or involve some combination of the two. It is important to note that both risks and benefits are vital to any accurate assessment. For example, in the area of risks, even a technology that is considered safe in environmental or human health terms may be more complex from the perspective of international trade and commerce.

Similarly, in the area of benefits, liberal biosafety regimes will promote the development of a base of scientific skills that can independently inform risk assessment as well as actually conduct applied research. As a result, in some areas of biotechnology policy, one may actually need to focus on incentives more than burdens. Quite apart from scientific complexity, biotechnology policy is surrounded by a sometimes bewildering array of connected fields and their associated regulatory frameworks. For example, parties to the Cartagena Protocol are, almost invariably, also parties to the World Trade Organisation's Agreement on Sanitary and Phytosanitary Standards and should, therefore, seek to reflect an appropriate blend of these different commitments in their policies.

The relationship between agrobiodiversity and modern biotechnologies is typical of the complexities described above. There is enormous potential for synergies, with the diversity of plant, animal and microbial life providing the most effective source of building blocks for modern biotechnologies, and these technologies, in turn, significantly broadening the options for the conservation of agrobiodiversity. However, agrobio-diversity may also be placed at risk by poor management of the environmental or commercial implications of some techno-logies. The development of long-term sustainable biotechnology strategies should be directly linked with the expression of coherent policies and supporting regulatory structures that genuinely reflect political objectives and realities.

\section{Conclusion}

Most West African countries individually have limited capacities and face enormous constraints in genetic resources collection, conservation and deployment for use in promoting human wellbeing. Until these fundamental constraints are resolved, efforts to make the best use of biotechnology to promote agriculture in West Africa will be severely hampered by ongoing loss of genetic materials and lack of knowledge of the potential of available biological resources. On the other hand, biotechnology tools offer opportunities for conserving genetic resources. West African countries have individually attempted to set up genetic resources conservation units without much success due to human, infrastructural and maintenance constraints. The conference of West and Central Africa Research Directors (WECARD/CORAF), as a regional research arm

West African Journal of Applied Ecology, Vol. 12, 2008 
of the West African Economic Community (ECOWAS), is promoting the development of selected functional conservation infrastructure sites and their personnel as Nodal Centres of Excellence to serve the West and Central Africa region in the conservation and use of genetic resources. It would be strategic for ECOWAS to establish and explore beneficial linkages between the WECARD/CORAF initiative and biotechnology programmes within the sub-region.

In exploring how best to manage the strategic interplay between biotechnology and diversity in agricultural biodiversity resources, there is need to address a few pertinent questions. How can biotechnology be used to enhance access to or management (conservation and use) of genetic resources? Which biotechnology tools are relevant to each country's situations? What alternatives to bio-technology are available to effectively achieve similar ends? What are the resource implications (human, equipment, operating expenses) of sustaining a biotechnology programme vis-à-vis the alternative methods for conservation of agricultural biological resources? What is there to lose if biotech-nology investment for the conservation of genetic resources is not made?

Investment in developing and maintaining functional biotechnology capabilities (human, material, etc.) is very expensive. It would not be strategic for each ECOWAS member country to invest scarce resources in setting up the full range of biotechnology capabilities. Developing and maintaining a few existing biotechnology centres to provide regional services, within the ECOWAS framework, could be an attractive alternative. There is need to decide how to mobilize political will to face the common problems that can be solved using biotechnology. It is important to provide adequate information at all levels (political, extension, farmers, etc.) to inform decisions and choices based on the real value and potential risks of biotechnology.

References
AU/NEPAD High-Level Panel on Biotechnology (2006). Freedom to Innovate, Biotechnology in Africa's
Development. Draft Report of the High-Level African Panel on Modern Biotechnology of the African Union (AU)
and the New Partnership for Africa's Development http://www.nepadst.org/doclibrary/pdfs/abp_july2006.pdf.

Cox D. (1988). Protection of Plant Genetic Resources. Echo Dev. Notes 61.

Devos K. M. and Gale M. D. (2002). Genome relationships: the grass model in current research. Pl.Cell. 12: 637-646.

Englemann F. (2004). Plant cryopreservation: progress and prospects. In-vitro Cellular and Development Biology. Plant. 40(5): 427-433.

FAO (1993). International Technical Workshop jointly organized by the Food and Agriculture Organization of the United Nations and the Secretariat of the Convention on Biological Diversity (SCBD), with support of the Government of the Netherlands, 2-4December. www.fao.org/sd/epdirect/EPre0063.htm

FAO (1999). Background Paper 1: Agricultural Biodiversity. FAO/Netherlands Conference on the Multifunctional Character of Agriculture and Land. Maastricht.

Helliot B., Panis B., Poumay Y., Swennen R., Lepoivre P. and Frison E. (2002). Cryopre-servation for the elimination of cucumber mosaic and banana streak viruses from banana (Musa spp.). Pl. Cell Rep. 20(12): 11171122.

Mulvany P. (1998). Agricultural Biodiversity. Connections. The UNED-UK Newsletter. http://www.ukabc.org/uneduk2.htm

Panis B., Strosse H., Remy S., Sági L. and Swennen R. (2004). Cryopreservation of banana tissues: support for germplasm conservation and banana improvement. In Banana Improvement: Cellular, Molecular Biology, and Induced Mutations. (M. Jain and R. Swennen, ed.). Science Publishers. Enfield (NH).

\section{INSTRUCTIONS TO AUTHORS}

Papers for submission to the West African Journal of Applied Ecology should be written in English and should not exceed 8,000 words in total length. Papers should not have been submitted or be considered for submission for publication elsewhere. Ideas expressed in papers

West African Journal of Applied Ecology, Vol. 12, 2008 
that are published in the Journal are entirely those of the authors and not those of the Editors. Papers submitted will be subjected to peer review before possible publication. Authors are encouraged to suggest names of two potential referees. They should, however, not be from the same Institute, nor should they have been at any time closely associated professionally with the author(s) of the paper being submitted. The Editorial Board regards these names as suggestions only, and is not obliged to use any or both of the proposed names.

Papers for publication should be submitted to:

The Editor

West African Journal of Applied Ecology

Ecological Laboratory Unit

University of Ghana

P. O. Box LG59

Legon, Accra

Ghana.

\section{Reprints}

Authors will receive one copy of the Journal in which their papers are published. Author(s) may order reprints at their own cost. Proofs come to the authors with reprints order forms.

\section{Copyright}

Authors publish in the Journal with the understanding that they assign their copyright to the West Africa Journal of Applied Ecology.

\section{Organization of Manuscripts}

Authors should please submit three copies of their manuscript, typed with double spacing, including figure legends, table footnotes and references, on one side of A4 $(30 \mathrm{~cm} \times 21 \mathrm{~cm})$ size high quality paper, with $2.5 \mathrm{~cm}$ margins on all four sides. Papers must be numbered sequentially, including the abstract, figure legends and tables. The paper should be organised into the following sections:

- Title page, indicating title, short running title, name of each author, and, for each author, position held and full address. Please, indicate which address should be used for correspondence and reprints requests where there is more than one author.

- Abstract, not exceeding 300 words. Should provide a brief summary of the basic content of the paper, including major methods used, results and deductions or conclusions. Avoid abbreviations and do not include tables and diagrams, and, unless absolutely necessary, references should be excluded.

- Introduction, to supply background information and references of earlier published reports related to the study, and provide the rationale for undertaking the study. Should avoid excessive references, particularly those not closely related to the study.

- Materials and methods, to provide sufficient technical information on approaches, materials and equipment, etc., used to obtain the results reported, such that the work could be duplicated by someone else.

- Results, reporting only the results of the experiments, illustrated by Tables and Figures.

- Discussion, an interpretation of the results, and its relation to other previously published work on the subject, and must not be a recapitulation of results.

- Acknowledgement, indicating the source of any financial support or personal assistance received in carrying out the study.

West African Journal of Applied Ecology, Vol. 12, 2008 
- References, listing all quoted published material, arranged in alphabetical order, typed doublespace. Abbreviate names of journals according to BIOSIS Serial Sources (Bio-Sciences Information Service, Philadelphia, Pa., 1997).

Follow the styles shown in the examples below:

Bhuvaneswari T. V. and Selheim B. (1985). Root hair deformation in the white clover/Rhizobium trifolii, symbiosis. Physiol. Plant. 63: 25-34.

Deutscher M. P. (1990). Guide to Protein Purification. Academic Press Inc., San Diego, USA.

Dreyfs B., Garcia J. L. and Gillis M. (1988). Characterization of Azorhizobium caulinodans gen. Nov., sp. Nov., a stem-nodulating nitrogen-fixing bacterium isolated from Sesbania rostrata. Int. J. Syst. Bacteriol. 38: 89-98.

Van Rhee J. E. (1963). Earthworm activities and the breakdown of organic matter. In Soil Organisms. (J. Doeksen and J. van der Drift, ed.), pp. 55-59. North Holland, Amsterdam.

Sambrook J., Fritsch E. F. and Maniatis T. (1989). Molecular Cloning: A Laboratory Manual. Cold Spring Harbor Laboratory, Cold Spring Harbor, New York, USA.

References in the text should be quoted by the author's name and date in parenthesis and presented in date order. Where there are more than two authors, the first author only should be quoted, followed by et al. and date in parenthesis.

Illustrations and Tables, should be of high quality, and line drawing. Should be in a form suitable for reproduction without modification. Figures, photographs and tables should be added separately, i.e. not inserted in the typed text, with each figure or table presented on a separate page, and designed for linear reduction in the ratio of $2: 1$ or $3: 2$, for printing within a column width of $6.5 \mathrm{~cm}$ or a double-column width of $13.6 \mathrm{~cm}$. Lettering on axes and within figures should be clear and big enough to be legible even upon 50-75\% reduction in size. The figure legends should be typed, correspondingly, on a separate page, headed Legends, at the end of the article. The author's name, the figure number and indication of its proper orientation should be written lightly on the back of each figure. Photographs should be supplied as blackand-white high, contrast glossy prints. Colour plates may be inserted only if author agrees to pay for the expenses involved.

\section{Units}

All measures should be indicated in SI units. Where necessary they may be followed by the equivalent in traditional units in parenthesis.

\section{Change of Address}

Authors should promptly notify the Editor of any change of address.

\section{Reviews of Books}

Books and monographs will be reviewed by invited reviewers. They may be sent to the Editor.

\section{Notes to Authors}

- Copyright material. Authors have the responsibility of ensuring that co-authors are in agreement with the form and submission of the manuscript, and for obtaining permission to quote material from copyright sources.

- Returning of manuscripts to authors. Manuscripts submitted will not be returned to authors unless specifically requested at the time of submission. 
- Proofs will be sent to authors for final correction. However, authors are not expected to make extensive modifications of manuscripts at this stage, otherwise they would be surcharged. Proofs sent to authors for correction should be returned within a week of receipt, together with any order for reprints.

- Acceptance of manuscripts for publication. Acceptance of a manuscript for publication will be indicated explicitly in a letter to the authors, and whether any corrections or modifications are required to be made on the paper or not. Similarly, authors whose papers are rejected for publication will be notified. 